\title{
Aspectos tecnológicos do processamento de suco e fermentado alcoólico de caqui
}

\author{
RESUMO
}

Aline Alberti

alinealberti@gmail.com

http://orcid.org/0000-0002-9787-4630

Programa de Mestrado em Ciência,

Tecnologia e Engenharia de Alimentos,

Departamento de Engenharia de Alimentos,

Universidade Estadual de Ponta Grossa, Campus Uvaranas, Ponta Grossa, Paraná
Brasil.

Amanda Alves Prestes

aprestes04@gmail.com

Programa de Mestrado em Ciência,

Tecnologia e Engenharia de Alimentos,

Departamento de Engenharia de Alimentos,

Universidade Estadual de Ponta Grossa,

Campus Uvaranas, Ponta Grossa, Paraná, Brasil.

Giuliane Sviercoski

giu.sviercoski@gmail.com

Programa de Mestrado em Ciência

Tecnologia e Engenharia de Alimentos,

Departamento de Engenharia de Alimentos,

Universidade Estadual de Ponta Grossa,

Brasil.

Acácio Antonio Ferreira Zielinski aczielinski@gmail.com

Departamento de Engenharia Química e de Engenharia Alimentos, Universidade Federa de Santa Catarina, Santa Catarina, Brasil.

\section{Alessandro Nogueira}

\section{alessandronog@yahoo.com.br \\ https://orcid.org/0000-0001-7671-3056}

Programa de Mestrado em Ciência,

Tecnologia e Engenharia de Alimentos,

Departamento de Engenharia de Alimentos,

Universidade Estadual de Ponta Grossa,

Campus Uvaranas, Ponta Grossa, Paraná, Brasil.
A classificação comercial do caqui pode gerar até $40 \%$ de descarte. Estas frutas são pouco aproveitadas devido à falta de informações tecnológicas que possibilitem a sua transformação em produtos com alto valor agregado. O objetivo do trabalho consistiu em avaliar a extração de suco/mosto e os parâmetros de fermentação alcoólica de caqui das cultivares Fuyu (Amagaki) e Kakimel (Shibugaki). O rendimento de extração do suco/mosto foi avaliado em frutos maduros após os seguintes tratamentos [1] trituração e prensagem; [2] trituração, maceração (Pectinex 3XL e Ultrazym AFP-L) e centrifugação; e nos frutos em fase de senescência, com esmagamento manual e maceração enzimática. Análises físicoquímicas foram realizadas nos sucos/mostos e nos fermentados de caqui. Os maiores rendimentos de 53 e $40 \%$ foram obtidos após trituração dos frutos seguida de uma liquefação enzimática (Ultrazym AFP-L) e centrifugação, para os caquis Fuyu e Kakimel, respectivamente. O mosto/suco despectinizado do caqui Fuyu apresentou coloração levemente amarelada, ao contrário do caqui Kakimel com coloração amarelo dourado. Os teores de açúcar (maior que 17,4 g/100mL) e de nitrogênio (maior que $219 \mathrm{mg} / \mathrm{L}$ ) do mosto, contagem de leveduras maior que 3,0 × $10^{7}$ células $/ \mathrm{mL}$, o tempo da fermentação alcoólica (9,5-10,5 dias) e o grau alcoólico final $\left(9^{\circ} \mathrm{GL}\right)$ indicam que o caqui apresenta aptidão para elaboração de bebidas alcoólicas. Portanto, este trabalho fornece informações tecnológicas sobre o processamento de bebidas a partir de caqui de descarte.

PALAVRAS-CHAVE: rendimento, maceração enzimática, despectinização, fermentação alcoólica. 


\section{INTRODUÇÃO}

O caqui (Diospyros kaki L.), originário da Ásia, apresentou em 2016, uma produção mundial de 5,4 milhões de toneladas (FAOSTAT, 2018). A China, maior produtor mundial de caqui, nesse mesmo ano produziu 3,9 milhões de toneladas, seguido pela Coreia do Sul, com 405,7 mil toneladas e pelo Japão, com 232,9 mil toneladas. O Brasil é o quarto maior produtor mundial de caqui, com $161 \mathrm{mil}$ toneladas (FAOSTAT, 2018).

No Brasil, o cultivo do fruto foi iniciado por imigrantes japoneses no início do século XX (FERRI et al., 2002). O interesse por essa cultura justifica-se por sua ótima adaptação às condições edafoclimáticas de algumas regiões brasileiras (GONZALES; AYUB; WERLANG, 2005). Na Região Sudeste, concentram-se os maiores Estados produtores do fruto, com destaque para São Paulo responsável por $80 \%$ da produção total do país (IBGE, 2016).

Os caquis são classificados de acordo com a aparência e composição do fruto. Aqueles que apresentam elevado teor de taninos na polpa, com ou sem sementes, são denominados "Shibugaki", enquanto os não taninosos, de polpa amarela, "Amagaki" (CAMPO DALL'ORTO et al., 1996).

A cada safra brasileira, aproximadamente $40 \%$ da produção de caqui, destinada ao comércio in natura, é descartada na classificação comercial devido a defeitos fitopatológicos (alta perecibilidade), ao tamanho fora do padrão e formatos irregulares. A maior parte do descarte apresenta qualidade para ser transformado em produtos como sucos/polpas, fermentados alcoólicos, vinagres e caquis desidratados agregando valor a estas frutas (PÉREZ-BURILLO et al., 2018). Entretanto, o processo industrial é pouco difundido quando comparado com outras frutas (UBEDA et al., 2011; IMAl et al., 2001).

Portanto, o objetivo desse trabalho baseou-se em avaliar diferentes métodos de extração e obtenção do suco/mosto, rendimento e parâmetros de fermentação dos mostos de duas cultivares de caqui, Fuyu e Kakimel.

\section{MATERIAIS E MÉTODOS}

\section{MATERIAIS}

As cultivares de caqui Fuyu e Kakimel foram adquiridos em pomares localizados na cidade de Curitiba, Paraná, Brasil. Os frutos de ambas as cultivares apresentavam-se maduros com cor laranjada predominante (>90\%) (KRAMMES et al., 2005).

Os reagentes químicos utilizados foram de pureza pró-análise. O complexo enzimático de maceração Ultrazym AFP-L (pectina liase, celulase e poligalacturonase) e a enzima pectinolítica Pectinex 3XL (pectina transeliminase, poligalacturonase e pectina esterase), foram cedidos pela LNF de Bento Gonçalves (RS) (Novozymes, Alemanha).

Os equipamentos, utensílios e consumíveis foram fornecidos pelos Laboratórios do Curso de Engenharia de Alimentos da Universidade Estadual de 


\section{PROCESSAMENTO DO SUCO/MOSTO}

As frutas foram selecionadas sendo descartadas as que apresentavam defeitos fitopatológicos acima de $20 \mathrm{~mm}$ de diâmetro e incidências menores foram retiradas das frutas com auxílio de uma faca. O cálice das frutas foi retirado manualmente, a fim de aumentar a eficiência da lavagem e da sanitização na redução da população microbiota. As frutas foram sanitizadas em solução de 100 $\mathrm{mg} / \mathrm{L}$ de cloro ativo por um período de 15 minutos à $10^{\circ} \mathrm{C}$, e em seguida enxaguadas em água potável e processadas. Quatro protocolos foram utilizados, sendo codificados como " $A$ ", " $B$ ", " $C$ " e " $D$ ". No primeiro, denominado de " $A$ ", os caquis, foram triturados em multiprocessador de alimentos (Metvisa, Tipo MPA, Brasil) e a massa obtida foi acondicionada em telas de plástico poroso de grau alimentício (diâmetro do fio 33 BWG, abertura de $1 \mathrm{~mm}^{2}$, Vonder) que, superpostos, foram submetidos a uma pressão de $3,0 \mathrm{kgf} / \mathrm{cm}^{2}$ (Prensa hidráulica Eureka, Hoppe Ind. Ltda, Brasil) durante cinco (5) minutos. O rendimento foi avaliado nos sucos integrais e o suco bruto despectinizado com uso de enzimas Pectinex 3XL, a uma proporção de $3 \mathrm{~mL} / \mathrm{hL}$, sendo mantido a 40 으 durante 60 minutos e, após sedimentação a frio $\left(10^{\circ} \mathrm{C}\right)$, o sobrenadante foi trasfegado, engarrafado e conservado à $4^{\circ} \mathrm{C}$. Amostras das duas cultivares foram trituradas em multiprocessador e a massa obtida foi dividida em dois lotes a fim de avaliar a liquefação enzimática. No segundo tratamento, denominado " $B$ ", diferiu-se do tratamento " $A$ " pela realização de um processo de liquefação enzimática (enzima Pectinex $3 \mathrm{XL}$ ) a uma proporção de $3 \mathrm{~mL} / 100 \mathrm{~kg}$, a $45^{\circ} \mathrm{C}$ durante 60 minutos. Em sequência, no tratamento denominado " $\boldsymbol{C}$ " foi adicionada de enzima Ultrazym AFP-L, no processo de liquefação, a uma proporção de $0,9 \mathrm{~mL} / 100 \mathrm{~kg}$, a 30 으 por 12 horas. Após o período de maceração, as amostras foram centrifugadas a 1200 rpm $(200 \mathrm{~g})$ durante 5 minutos (Centrifuga Fanem Ltda, Modelo 214, Brasil), engarrafadas e conservadas à $4^{\circ} \mathrm{C}$. No quarto tratamento, " $\boldsymbol{D}$ ", os frutos foram esmagados manualmente e, após a maceração com enzima de liquefação Ultrazym a uma proporção de $0,9 \mathrm{~mL} / 100 \mathrm{~kg}\left(30^{\circ} \mathrm{C}\right.$ por 12 horas), o suco foi separado por centrifugação. Na Figura 1, é apresentado o diagrama de blocos do processamento dos sucos produzidos.

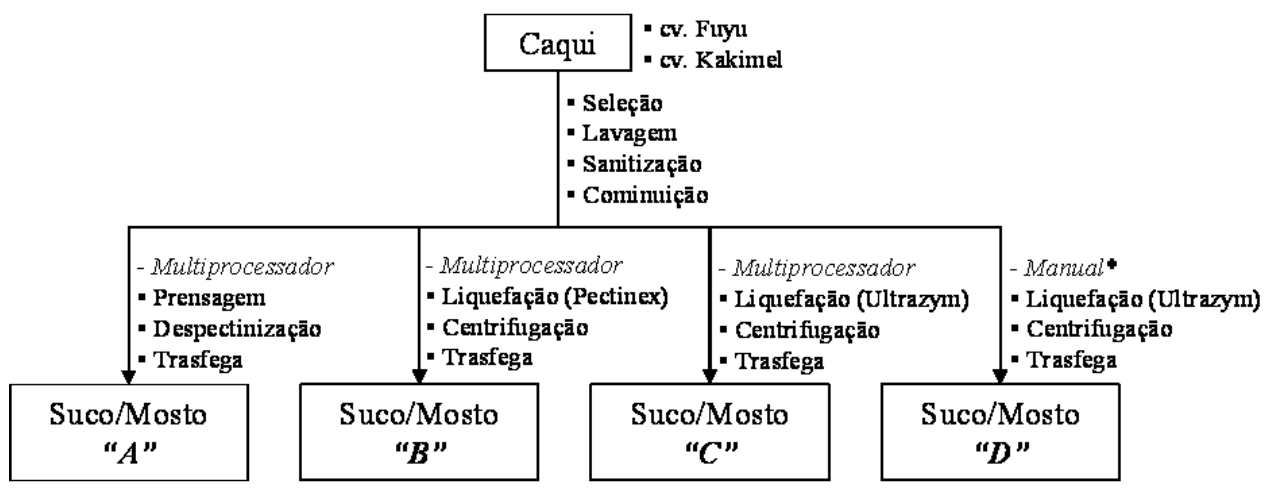

Figura 1. Fluxograma do processamento de suco/mosto de caqui. Nota: ${ }^{(*)}$ processo manual foi realizado apenas para a cultivar Fuyu em estádio de senescência. 


\section{CINÉTICA DE FERMENTAÇÃO}

Para a cinética de fermentação, os mostos da cultivar Fuyu, obtidos por diferentes extrações, foram homogeneizados assim como para a cultivar de Kakimel. Estes mostos foram divididos em erlenmeyers de $500 \mathrm{~mL}$, munidos de batoque, onde ocorreu o processo fermentativo. Em seguida foram adicionados 20 $\mathrm{g} / \mathrm{hL}$ de levedura seca ativa (LSA) Saccharomyces cerevisiae (Uvaferm CK, Danstar Ferment GAC, Dinamarca), previamente hidratada conforme recomendação do fabricante. Este inóculo corresponde a uma população inicial de 1,0-2,0 × $10^{6}$ células $/ \mathrm{mL}$.

A fermentação foi conduzida em temperatura de $20 \pm 2{ }^{\circ} \mathrm{C}$ durante 17 dias ou 408 horas (tempo de fermentação). 0 monitoramento da fermentação transcorreu nos tempos zero, 24, 48, 72, 96, 216 e 408 horas. Ao final de cada tempo, o mosto em fermentação foi centrifugado a 2000 rpm (Centrifuga Fanem Ltda, Modelo 214, Brasil) durante 20 minutos sendo em seguida trasfegado, engarrafado e armazenado a $-18^{\circ} \mathrm{C}$.

$\mathrm{O}$ processo foi monitorado pela perda de massa dos fermentadores devido à liberação de gás carbônico $\left(\mathrm{CO}_{2} \mathrm{~g} / \mathrm{L}\right)$. A partir destes dados foi calculada a velocidade máxima $\left(\mathrm{dCO}_{2} / \mathrm{dt}\right.$ ) em dias (BELY; SABLAYROLLES; BARRE, 1990; ALBERTI et al., 2011).

\section{ANÁLISE FÍSICO-QUÍMICA}

$\mathrm{O}$ pH foi determinado em potenciômetro digital (HANNA Instruments, modelo HI 84249, Brasil). Os teores de etanol foram determinados por ebuliometria em ${ }^{\circ} \mathrm{GL}$ (CURVELO-GARCIA, 1980). Os teores de açúcares redutores totais foram determinados pelo método de Somogyi-Nelson (TANNER; BRUNNER, 1985). A acidez total titulável foi determinada pela neutralização com $\mathrm{NaOH} 0,1 \mathrm{~N}$ até $\mathrm{pH}$ $8,33 \mathrm{com}$ fenolftaleína e os resultados foram expressos em $\mathrm{g} / 100 \mathrm{~mL}$ de ácido málico (IAL, 2008). A acidez volátil foi determinada por titulação após volatilização conforme método do Instituto Adolfo Lutz (IAL, 2008). O nitrogênio total foi quantificado pelo método de Kjedhal (IAL, 2008).

\section{CONTAGEM DE LEVEDURAS}

A contagem de células viáveis da $S$. cerevisiae foi realizada em câmara de Newbauer (XB-K-25, SMIC, China), pelo método de contagem direta em microscópio óptico, utilizando o corante azul de metileno (BONNEU et al., 1991).

\section{ANÁLISE ESTATIÍSTICA}

Os resultados analisados foram expressos como média e desvio padrão amostral. A homogeneidade das variâncias foi verificada pelo teste de Levene e as diferenças significativas $(p<0,05)$ dos valores médios foram analisados utilizando ANOVA fator único. $O$ teste de Fisher LSD foi utilizado para comparação de médias, com nível de significância de $(p>0,05)$. Todos os dados foram analisados através do Software Statistica, 13.2. (StatSoft, USA). 


\section{RESULTADOS E DISCUSSÃO}

\section{EXTRAÇÃO DO SUCO/MOSTO}

As frutas utilizadas nos experimentos ' $\mathbf{A}$ ', 'B' e 'C' estavam em estádio de maturação pleno com frutos de coloração superficial laranja, ideal para consumo in natura e para o processamento industrial (KRAMMES et al., 2005). Entretanto, o experimento ' $D$ ' com esmagamento manual (processo utilizado por pequenos produtores) foi realizado para as frutas em estádio de senescência (coloração superficial vermelha), sendo efetivo apenas para a cultivar Fuyu. Em geral, a cultivar Fuyu apresentou melhores rendimentos em relação a cultivar Kakimel. Apenas no tratamento 'B' o rendimento da Fuyu não foi superior (Figura 2), indicando que as enzimas pectinolíticas têm pouca influência no processo de maceração desta cultivar. Os tratamentos com maiores rendimentos de suco/mosto, aproximadamente duas vezes superior ao tratamento ' $A$ ', foram para os tratamentos ' $C$ ' e ' $D$ ' (Figura 2). Desta forma, a adição da Ultrazym apresentou o melhor efeito sobre a hidrólise da parede celular dos caquis, possivelmente devido ao blend e atividade das enzimas pectinaesterase, poligalacturonase e celulase (MACEDO et al., 2015). Estes resultados foram superiores aos encontrados por Oliveira et al. (2006) que conseguiram um aumento do rendimento de $18,5 \%$ de suco de maçãs no processo de maceração quando comparado ao processo de prensagem.

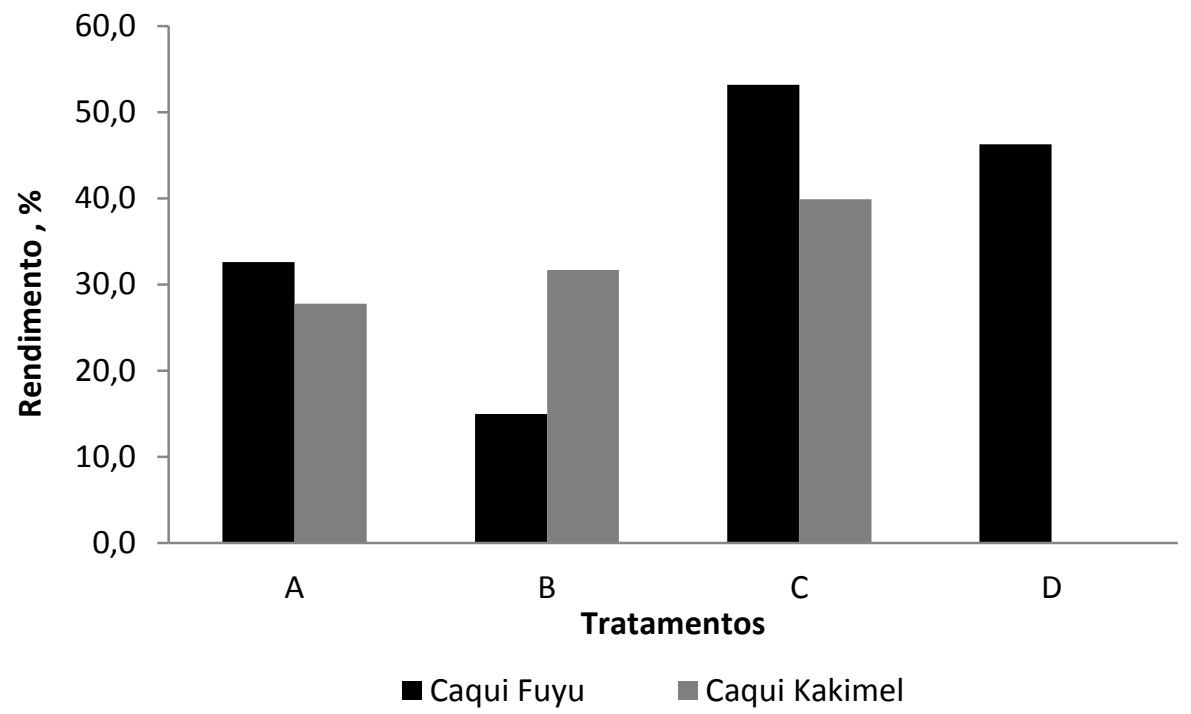

Figura 2. Efeito do processamento $(\mathbf{A}=$ triturado e prensado, $\mathbf{B}=$ triturado $\mathrm{e}$ liquefeito (Pectinex $3 \mathrm{XL}$ ), $\mathbf{C}=$ triturado e liquefeito (Ultrazym) e $\mathbf{D}=$ esmagado manualmente e liquefeito (Ultrazym) no rendimento de suco/mosto de caqui Fuyu e Kakimel.

Os sucos/mostos obtidos do tratamento ' $A$ ' foram tratados com enzima pectinolítica, uma vez que o caqui apresenta elevadas concentrações de pectina gerando um suco com elevada viscosidade prejudicando operações de escoamento e de filtrações (Figura 3). A concentração da enzima e as condições foram às mesmas utilizadas em suco de maçã, sendo eficiente em ambas as cultivares. Entretanto, os sucos/mostos após a despectinização e filtração, 
apresentaram diferença de coloração (Figura 3). O Kakimel contém elevado teor de taninos insolúveis (Shibugaki), como catequina, epicatequina, epicatequina galato e epigalocatequina galato (PÉREZ-BURILLO et al., 2018). Esses compostos são substratos da enzima polifenoloxidase (PPO) e os elevados teores presentes nesse cultivar desencadeiam, durante o processamento, a reação de escurecimento enzimático que resulta em um suco de coloração amarelo dourado escuro (Figura 3) (KIEWNING; WOLLSEIFEN; SCHMITZ-EIBERGER, 2013). Na China o suco integral de caqui, principal forma de consumo, apresenta coloração amarelolaranjada, porém o suco despectinizado pode variar de uma coloração amareloclara ao amarelo-escuro, podendo ser varietal ou elaborado com blends de cultivares. A cultivar Fuyu, caracterizada como um fruto não adstringente (Amagaki), apresenta baixos teores de taninos, resultando em uma cor amarelo clara devido à reação de escurecimento enzimático não ser tão intensa como na cultivar Kakimel.

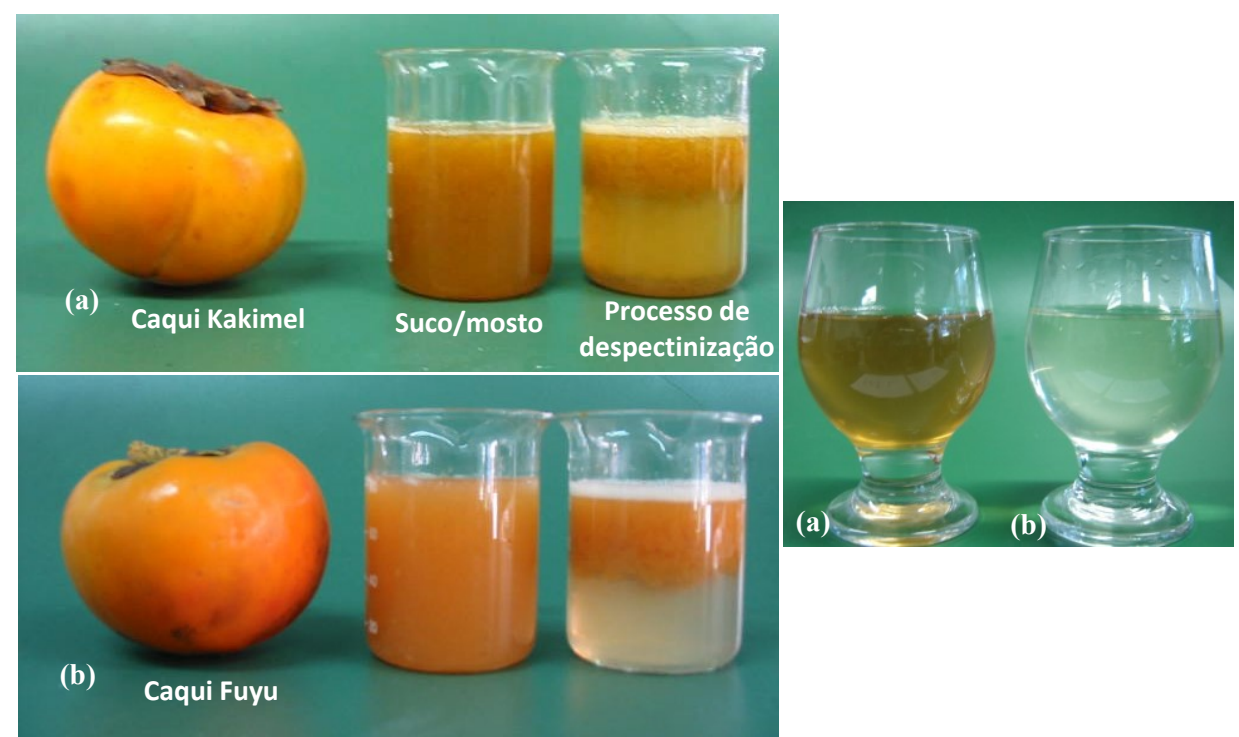

Figura 3. Características do suco/mosto de Kakimel (a) e Fuyu (b) recém-extraído, do processo de despectinização e da coloração das bebidas após a filtração.

Segundo Gorinstein et al. (2001), o caqui apresenta um teor de açúcar total entre 14 a $24 \mathrm{~g} / 100 \mathrm{~g}$ de fruta fresca. Dentre os principais açúcares encontrados na composição do caqui estão a glucose, frutose e sacarose. Em geral, a glucose está presente em maiores concentrações $(4,78-8,79 \mathrm{~g} / 100 \mathrm{~g})$, seguida pela frutose $(3,8$ $7,78 \mathrm{~g} / 100 \mathrm{~g}$ ) e sacarose $(0,9-1,2 \mathrm{~g} / 100 \mathrm{~g}$ ) (NOVILLO et al., 2016; PÉREZ-BURILLO et al., 2018; VERBERIC et al., 2010). A cultivar, tratos culturais, clima e local geográfico são fatores que podem influenciar na concentração de açúcares do fruto (GIORDANI; DOUMETT; NIN; BUBBA, 2011). O teor elevado de açúcares enaltece a qualidade do fruto, assim como os produtos obtidos a partir do mesmo. As cultivares de caqui estudadas, apresentaram uma diferença significativa de $4 \%$ no teor de açúcar total do suco/mosto (Tabela 1 ).

O teor de nitrogênio nos mostos de caqui está relacionado ao conteúdo de aminoácidos e peptídeos presentes, podendo variar devido ao efeito varietal, estádio de maturação, tratos culturais, idade do pomar e adubações no solo de cultivo (KUNEMAN; BRADDOCK; McCHESNEY, 1988; JIMÉNEZ-SÁNCHEZ et al., 
2015). Entre as duas cultivares de caqui, o teor de nitrogênio no suco/mosto apresentou diferença significativa (Tabela 1). Callejón et al. (2015) observaram que o tratamento com enzimas pectinolíticas no mosto causava uma redução de nitrogênio disponível para fermentação, devido a decantação desse nutriente aderido aos componentes da parede celular hidrolisados. Entretanto, este efeito não foi avaliado neste trabalho. O nitrogênio, durante o processo fermentativo, é fundamental no metabolismo da levedura (crescimento e fermentação alcoólica) e formação de compostos secundários, sendo esses responsáveis pelo aroma e sabor da bebida.

Tabela 1. Parâmetros físico-químicos dos mostos e fermentados das cultivares de caqui Fuyu e Kakimel.

\begin{tabular}{|c|c|c|c|c|}
\hline \multirow{2}{*}{$\begin{array}{l}\text { Parâmetros } \\
\text { Analíticos }\end{array}$} & \multicolumn{2}{|c|}{ Fuyu } & \multicolumn{2}{|c|}{ Kakimel } \\
\hline & Mosto & Fermentado & Mosto & Fermentado \\
\hline $\mathrm{ART}^{*}(\mathrm{~g} / 100 \mathrm{~mL})$ & $18,12 \pm 0,31^{a}$ & $0,56 \pm 0,02^{c}$ & $17,40 \pm 0,46^{b}$ & $0,69 \pm 0,01^{c}$ \\
\hline Nitrogênio (mg/L) & $219,00 \pm 10,00^{d}$ & $99,00 \pm 1,00^{c}$ & $345,00 \pm 2,00^{a}$ & $234,00 \pm 2,00^{b}$ \\
\hline Acidez total $(\mathrm{g} / 100 \mathrm{~mL})$ & $0,17 \pm 0,01^{c}$ & $0,32 \pm 0,01^{b}$ & $0,17 \pm 0,12^{c}$ & $0,43 \pm 0,01^{a}$ \\
\hline $\mathrm{pH}$ & $3,95 \pm 0,01$ & $3,83 \pm 0,01$ & $3,73 \pm 0,01$ & $3,53 \pm 0,01$ \\
\hline Acidez volátil (mg/L) & $5,00 \pm 1,00^{c}$ & $12,00 \pm 1,00^{a}$ & $6,00 \pm 1,00^{b c}$ & $7,00 \pm 1,00^{b}$ \\
\hline
\end{tabular}

Nota: Resultados expressos com a média \pm desvio padrão. Letras diferentes na mesma linha indicam diferença significativa $(p<0,05)$ para análise de variância (ANOVA). *ART: Açúcares Redutores Totais.

Classificado como um fruto de baixa acidez, o caqui apresenta teores, em média, entre 0,16 - 0,23 g/100 $\mathrm{mL}$ em ácido málico. Dentre os principais ácidos orgânicos encontrados na composição desse fruto estão o ácido málico, ácido cítrico e ácido fumárico. $\mathrm{O}$ ácido málico, em maiores concentrações no fruto fresco $(83,9 \mathrm{mg} / 100 \mathrm{~g})$, seguido pelo ácido cítrico $(30,5 \mathrm{mg} / 100 \mathrm{~g})$ e ácido fumárico $(12,5$ $\mathrm{mg} / 100 \mathrm{~g}$ ) (VERBERIC et al., 2010; PÉREZ-BURILLO et al., 2018). Os mostos de caqui Fuyu e Kakimel obtidos, não apresentaram diferença significativa entre si para as análises de acidez total titulável e acidez volátil (Tabela 1).

\section{CINÉTICA DE FERMENTAÇÃO}

A população máxima de leveduras ficou entre 3 e $5 \times 10^{7}$ células $/ \mathrm{mL}$, nos fermentados de Fuyu e Kakimel, respectivamente (Figura 4A). Estes valores indicam que o caqui possui todos os nutrientes essenciais ao desenvolvimento da Saccharomyces cerevisiae (ALBERTI et al., 2011). As leveduras consumiram teores ao redor de $100 \mathrm{mg} / \mathrm{L}$ de nitrogênio total (Tabela 1) nos primeiros 2 a 3 dias, para o fermentado de Fuyu e Kakimel, respectivamente (Figura 4A). Os compostos nitrogenados são utilizados pela Saccharomyces sp. durante a fase de crescimento, exercendo um importante papel na síntese de proteínas, influenciando o crescimento e o metabolismo das leveduras (JULIEN et al., 2000). Segundo Alberti et al. (2011) as leveduras necessitam em mostos de maçãs de 75 a $150 \mathrm{mg} / \mathrm{L}$, estando de acordo com os valores observados neste trabalho. A velocidade de fermentação do fermentado de Fuyu foi maior comparado ao Kakimel, ainda que esta segunda cultivar apresente um maior teor de nitrogênio. Isto pode ser explicado pela maior intensidade da reação de escurecimento enzimático no 
Kakimel, a qual gerou um menor teor de oxigênio dissolvido no início da fermentação alcoólica. $O$ oxigênio dissolvido no mosto é essencial para a formação de ergosterol na membrana plasmática da levedura durante o seu crescimento (ALBERTI et al., 2014).
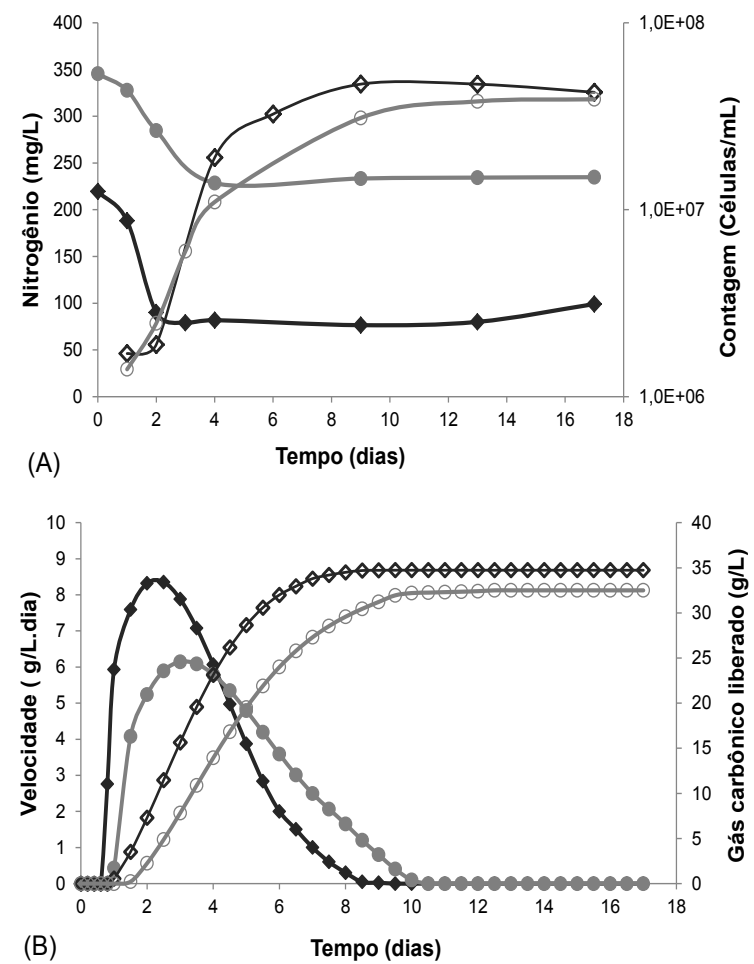

(B)

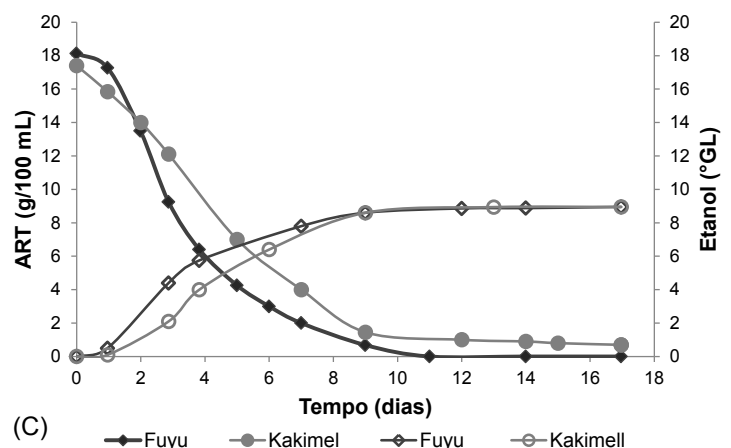

Figura 4. Curvas da cinética de fermentação: ' $A$ ': Curvas do consumo de nitrogênio e crescimento celular; 'B': Curvas de velocidade de fermentação e liberação de gás carbônico; ' $\mathbf{C}$ ': Curvas do consumo de açúcares redutores totais e produção de etanol.

Na Figura 4(B), observam-se as curvas cinéticas calculadas a partir da perda de massa dos fermentadores devido à liberação do gás carbônico durante a fermentação alcoólica, que possibilitou a geração de dados de velocidade de fermentação. O consumo dos açúcares fermentescíveis ocorreu até o oitavo e décimo dia de fermentação alcoólica, para o fermentado da cultivar Fuyu e Kakimel, respectivamente (Figura 4B). A fermentação tumultuosa gerou a formação de espuma atingindo $5-7 \%$ do volume útil dos fermentadores, para ambas as cultivares. Entretanto, ao final de 10 dias os fermentados apresentaram o mesmo teor alcoólico (Figura $4 \mathrm{C}$ ). 0 teor alcoólico de $9,0^{\circ} \mathrm{GL}$ indica que mais $89 \%$ 
dos açúcares eram fermentescíveis. Este teor alcóolico desempenha um papel importante na conservação do fermentado e seu teor apropriado na bebida, pode influenciar positivamente no sabor da mesma (WANG, ZHANG; 2014).

\section{CONCLUSÕES}

A trituração dos caquis seguido de maceração enzimática com Ultrazym AFPL foi mais eficiente para extração suco/mosto, com a obtenção de um maior rendimento. Uma vez que o conhecimento sobre bebidas à base de caqui é limitado, a composição química e a cinética de fermentação dos mostos do fruto indicam grande potencial para a elaboração de sucos e bebidas fermentadas, podendo ser aplicada com blend ou base na elaboração de novas bebidas e assim diminuir o desperdício dos frutos. Desta forma, o suco e o fermentado de caqui tem um grande potencial como novos produtos e novas alternativas ao consumidor.

\section{AGRADECIMENTOS}

Os autores agradecem ao CNPq, CAPES e Fundação Araucária pelas bolsas de estudo concedidas para a realização desse projeto, à LNF Bento GonçalvesNovozymes Latin America pelo fornecimento das enzimas utilizadas na elaboração. 


\title{
Technological aspects of the manufacture of juice and alcoholic fermented of persimmon
}

\begin{abstract}
Commercial classification of the persimmon can generate up to $40 \%$ of discard. These fruits are little used due to the lack of technological information that allows their transformation into products with high added value. The aim of this work was to evaluate the extraction of persimmon juice and the alcoholic fermentation of persimmon must of the cultivars Fuyu (Amagaki) and Kakimel (Shibugaki). The extraction yield of the persimmon juice was evaluated in ripe fruits after the following treatments [1] crushing and pressing; [2] crushing, maceration (Pectinex $3 \mathrm{XL}$ and Ultrazym AFP-L) and centrifugation; and in fruits in the senescence phase, with manual crushing and enzymatic maceration. Physicochemical analyzes were performed in the persimmon juices and alcoholic fermented. The highest yields of 53 and $40 \%$ were obtained after fruit crushing followed by an enzymatic maceration (Ultrazym AFP-L) and centrifugation for Fuyu and Kakimel persimmons, respectively. The despectinized juice of the Fuyu persimmon showed a light yellow color, unlike the Kakimel persimmon with a golden yellow color. Sugar content (> $17.4 \mathrm{~g} / 100 \mathrm{~mL})$ and nitrogen $(>219 \mathrm{mg} / \mathrm{L})$ of the must, yeast count $>3.0 \times 10^{7}$ cells $/ \mathrm{mL}$, alcoholic fermentation time (9.5-10, 5 days) and the final alcoholic degree (9 $\mathrm{OGL}$ ) indicate that the persimmon has aptitude for alcoholic beverages. Therefore, this paper provides technological information on the processing of beverages from discarded persimmon.
\end{abstract}

KEYWORDS: yield, enzymatic maceration, despectinization, alcoholic fermentation. 


\section{REFERÊNCIAS}

ALBERTI, A.; VIEIRA, R. G.; DRILLEAU, J. F.; WOSIACKI, G.; NOGUEIRA, A. Apple wine processing with different nitrogen contents. Brazilian Archives of Biology and Technology, v. 3, n. 54, p. 551 - 558, 2011.

https://dx.doi.org/10.1590/S1516-89132011000300017

ALBERTI, A.; BRAGA, C. M.; JASTER, H.; NOGUEIRA, A. Dissolved oxygen content in apple must: technological implications in cider processing. Journal of the Institute Brewing, v. 120, p. 65-70, 2014. doi:10.1002/jib.113

BELY, M.; SABLAYROLLES, J. M.; BARRE, P. Description of alcoholic fermentation kinetics: its variability and significance. American Journal of Enology and Viticulture. v. 41, p. 319-324, 1990.

<http://www.ajevonline.org/content/41/4/319>

BONNEU, M.; CROUZET, M.; URDACI, M.; AIGLE, M. Direct selection of yeast mutants with reduced viability on plates by eritrosine $b$ staining. Analytical Biochemistry, v. 193, p. 225-230, 1991.

<https://www.sciencedirect.com/science/article/pii/000326979190013J >

CALLEJÓN, R. M.; UBEDA, C.; HIDALGO, C.; MAS, A.; TRONCOSO, A. M.; MORALES, $M$. L. Changes on free amino acids during the alcoholic fermentation of strawberry and persimmon. International Journal of Food Science \& Technology, v. 50, n. 1, 48-54, 2015.

CAMPO DALL'ORTO, F.A.; OJIMA, M.; BARBOSA, W.; ZULLO, M.A.T. Novo processo de avaliação da adstringência dos frutos no melhoramento do caquizeiro. Bragantia v.55, p. 237- 243,1996.

<http://www.scielo.br/scielo.php?pid=S000687051996000200005\&script=sci_ab stract\&tlng=pt $>$

CURVELO-GARCIA, A. S. Controle de qualidade dos vinhos - química enológica métodos analíticos. Instituto da Vinha e do Vinho, Lisboa, 420p., 1988.

ELIAS; N. F.; BERBERT, P. A.; MOLINA, M. A. B.; VIANA, A. P.; DIONELLO, R. G.; QUEIROZ, V. A. V. Avaliação nutricional e sensorial de caqui cv fuyu submetido à desidratação osmótica e secagem por convecção. Ciência e Tecnologia de Alimentos, v. 28, n. 2, p. 322-328, 2008.< http://www.scielo.br/pdf/cta/v28n2/a09v28n2>

FAOSTAT, Food Agriculture Organization of the United Nations; Statistical database. Disponível em <http://www.fao.org/faostat/en/\#data/QC $>$, acesso em: 30 de jan. 2018. 
FERRI, V. C.; RINALDI, M. M.; LUCHETTA, L.; ROMBALDI, C. V. Qualidade de caquis Fuyu tratados com cálcio em pré-colheita e armazenados sob atmosfera modificada. Revista Brasileira de Fruticultura, v. 24, n. 2, p. 385-388, 2002. < http://www.scielo.br/pdf/rbf/v24n2/a22v24n2.pdf>

GILMAN, E.F.; WATSON, D.G. Diospyros kaki-japanese persimmon. Florida Cooperative Extension Service, Institute of Food and Agricultural Sciences, University of Florida, 1993.

GIORDANI, E.; DOUMETT, S.; NIN, S.; BUBBA, M.D. Selected primary and secondary metabolites in fresh persimmon (Diospyros kaki Thunb.): a review of analytical methods and current knowledge of fruit composition and health benefits. Food Research International. v 44, p. 1752-1767, 2011. < https://www.sciencedirect.com/science/article/pii/S0963996911000597>

GONZALEZ, A. F.; AYUB, R. A.; WERLANG, C. Controle da maturação de caqui cv. Fuyu tratado com aminoethoxyvinilglicina e armazenado a temperatura ambiente. Revista Brasileira de Agrociências, v.11, n.2, p.231 - 233, 2005. < https://periodicos.ufpel.edu.br/ojs2/index.php/CAST/article/viewFile/1215/1010 $>$

GORINSTEIN, S.; ZACHWIEJA, Z.; FOLTA, M.; BARTON, H.; PIOTROWICZ, J.; ZEMSER, M.; WEISZ, M.; TRAKHTENBERG, S.; MARTIN-BELLOSO, O. Comparative content of dietary fiber, total phenolics, and minerals in persimmons and apples. Journal of Agriculture and Food Chemistry, v. 49, p. 952-957, 2001. < https://pubs.acs.org/doi/abs/10.1021/jf000947k >

IAL - Instituto Adolfo Lutz. Métodos físicos e químicos para análise de alimentos. In: IAL. Normas Analíticas, Instituto Adolfo Lutz. São Paulo (SP): Brasil, 2008.

IMAI, K.; SHIOZAKI, S.; TSUDA, T.; NAKAO, Y.; OGATA, T.; HORIUCHI, S. Changes in sugar, organic acid contents and characteristics of kaki-shibu during fermentation. Journal of the Japanese Society for Horticultural Science, v. 70, n.1, p. 95-101, 2001.

INSTITUTO BRASILEIRO DE GEOGRAFIA E ESTATÍSITICA- IBGE; Sistema IBGE de Recuperação Automática - SIDRA;

Disponível em <https://sidra.ibge.gov.br/tabela/5457\#resultado> acesso em: 24 de out. 2017. 
polyphenols, sugars, and other polar compounds in persimmon juices produced under different technologies and their assessment in terms of compositional variations. Food Chemistry, v. 182, p. 282-291, 2015.

<https://www.sciencedirect.com/science/article/pii/S0308814615003593>

JULIEN, A.; ROUSTAN, J. L.; DULAU, L.; SABLAYROLLES, J. M. Comparison of nitrogen and oxygen demands of enological yeasts: technological consequences. American Journal of Enology and Viticulture, v. 51, n. 3, p. 215222, 2000. < http://www.ajevonline.org/content/51/3/215 >

KIEWNING, D.; WOLLSEIFEN, R.; SCHMITZ-EIBERGER, M. The impact of catechin and epicatechin, total phenols and PPO activity on the mald1 content in apple fruit. Food Chemistry, v. 140, p. 99-104, 2013. <https://www.sciencedirect.com/science/article/pii/S0308814613002045?via\%3 Dihub $\geq$

KRAMMES, J. G.; ARGENTA, L. C.; VIEIRA, M. J. Controle da maturação e conservação da qualidade pós-colheita de caqui 'Fuyu' pelo manejo do etileno. Revista Brasileira de Fruticultura, n.3, v. 27, p. 360-365, 2005. <https://dx.doi.org/10.1590/S0100-29452005000300006>.

KUNEMAN, D. W.; BRADDOCK, J. K.; MCCHESNEY, L. L. HPLC profile of amino acids in fruits juices as their (1-fluoro-2,4-dinitrophenyl)-5-I-alanine amide derivatives. Journal of Agricultural and Food Chemistry, v. 36, p. 6-9, 1988.

<https://pubs.acs.org/doi/pdf/10.1021/jf00079a002>

MACEDO, M.; ROBRIGUES, R. D. P.; PINTO, G. A. S.; DE BRITO, E. S. Influence of pectinolyttic and cellulotyc enzyme complexes on cashew bagasse maceration in order to obtain carotenoids. Journal of Food Science and Technology, n.52, v.6, p.3689-3693, 2015. doi:10.1007/s13197-014-1411-x.

NOVILLO, P.; SALVADOR, A.; CRISOSTO, C.; BESADA, C. Influence of persimmon astringency type on physico-chemical changes from the green stage to commercial harvest. Scientia Horticulturae, v. 206, p.7-14, 2016. <https://www.sciencedirect.com/science/article/pii/S0304423816302199>

OLIVEIRA, M. C. S. D.; SILVA, N. C. C.; NOGUEIRA, A.; WOSIACKI, G. Avaliação do método de liquefação enzimática na extração de suco de maçã. Ciência e Tecnologia de Alimentos, v. 26, p. 906-915, 2006.< http://www.scielo.br/pdf/cta/v26n4/29.pdf>

PÉREZ-BURILLO, S.; OLIVERAS, M. J.; QUESADA, J.; RUFIÁN-HENARES, J. A.; 
and kiwifruit. Food Research International, v. 105, p. 461-472, 2018 . <

https://www.sciencedirect.com/science/article/pii/S0963996917307949 >

TANNER, H.; BRUNNER, H. R. Getränke Anlytik - Untersuchungsmethode für dia Labor- und Betriebspraxis. Wädesnwill: Verlag Helles, 1985.

UBEDA, C.;HIDALGO, C.; TORIJA, M.J.; MAS, A.; TRONCOSO, A. M.; MORALES, M. L. Evaluation of antioxidant activity and total phenols index in persimmon vinegars produced by different processes. LWT-Food Science and Technology, v. 44, n. 7, p. 1591-1596, 2011. < https://www.sciencedirect.com/science/article/pii/S0023643811000764>

VEBERIC, R.; JURHAR, J.; MIKULIC-PETKOVSEK, M.; STAMPAR, F.; SCHMITZER, V. Comparative study of primary and secondary metabolites in 11 cultivars of persimmon fruit (Diospyros kaki L.). Food Chemistry, v. 119, p. 477-483, 2010. < <https://www.sciencedirect.com/science/article/pii/S0308814609008541>

WANG, J.M.; ZANG, J.X. Research progress on the origin and formation of aroma. China brewing, v. 5, p. 5-9, 2014.

WRIGHT, K. P.; KADER, A. A. Effect of controlled-atmosphere storage on the quality and carotenoid content of sliced persimmons and peaches. Postharvest Biology and Technology, v. 10, n. 1, p. 89-97, 1997.

<https://www.sciencedirect.com/science/article/pii/S0925521496000622>

Recebido: 16 fev. 2018.

Aprovado: 09 jan. 2019.

DOI: $10.3895 /$ rebrapa.v9n4.7780

Como citar:

ALBERTI, A. et al. Aspectos tecnológicos do processamento de suco e fermentado alcoólico de caqui. Brazilian Journal of Food Research, Campo Mourão, v. 9, n. 4, p. 74-87, out./dez. 2018. Disponível em:

https://periodicos.utfpr.edu.br/rebrapa

Correspondência:

Alessandro Nogueira

Programa de Mestrado em Ciência, Tecnologia e Engenharia de Alimentos, Departamento de Engenharia de Alimentos, Universidade Estadual de Ponta Grossa, Campus Uvaranas, Ponta Grossa, Paraná, Brasil.

Direito autoral: Este artigo está licenciado sob os termos da Licença Creative Commons-Atribuição 4.0 Internacional. 